\title{
Grid Diversity Operator for Some Population-Based Optimization Algorithms
}

\author{
Ahmed Salah \\ Faculty of Science, Mathematics Department \\ Mansoura University \\ Mansoura, Egypt \\ a_salah@mans.edu.eg
}

\author{
Emma Hart \\ Institute for Informatics and Digital Innovation \\ Edinburgh Napier University \\ Edinburgh, UK \\ e.hart@napier.ac.uk
}

\begin{abstract}
We present a novel diversity method named Grid Diversity Operator (GDO) that can be incorporated into multiple population-based optimization algorithms that guides the containing algorithm in creating new individuals in sparsely visited areas of the search space. Experimental tests on a set of unimodal and multimodal benchmark functions from the literature using GDO in conjunction with opt-aiNet algorithm show that GDO maintains better diversity in most cases, leading to an order-of-magnitude reduction in the number of objective function evaluations needed to converge while finding similar numbers of peaks in the majority of benchmarks.
\end{abstract}

\section{Categories and Subject Descriptors}

I.2 [Artificial Intelligence]: Problem Solving, Search

\section{Keywords}

Grid, Diversity, Optimization, Evolutionary Algorithms, Artificial Immune Systems

\section{INTRODUCTION}

Managing the diversity of a population has been recognized as one of the most influential factors within an Evolutionary Algorithms (EAs) right from their inception. From the exploration and exploitation perspective, an increase in diversity correlates with exploration phase of an optimization algorithm whilst a decrease correlates with the exploitation phase. Maintaining a diverse population through the use of exploration operators is key to achieving a balance between the two phases.

In this work we extend research within the field of diversity maintenance [1], proposing a novel genotypic diversity learning method named Grid-Diversity-Operator (GDO) that make use of the long-term history of all populations in order to suggest a biased distribution for new individuals. GDO is not specific to any particular algorithm, but can be used with any optimization algorithm that supports the use of infusion techniques, that is, through insertion of new individuals after a certain number of generations or through special initialization techniques are used.

This is the author's version of the work. It is posted here for your personal use. Not for redistribution. The definitive Version was published in GECCO 2015, http://dx.doi.org/ $10.1145 / 2739482.2764664$.

\section{GRID DIVERSITY OPERATOR (GDO)}

The Grid Diversity Operator (GDO) can be defined as a hybrid, non-niching, population-based, genotype diversity maintaining and learning technique. The basic idea is to split the feasible space (the domain) into smaller sub-spaces using the parameter $R \in \Re^{n}$, which defines the number of intervals per dimension, where $n$ is the number of dimensions for the problem. This process will form a $2 \mathrm{D}$ grid for 2dimensional problem etc. The GDO attempts to distribute new individuals to the grid slots that have received fewer visits over time, thus increasing the explorative power of the algorithm.

First, A memory archive is created as an empty dictionary that has $n$ components key, where each key matches a single value. The key components refer to the indices of a slot within the grid, while the value represents the number of individuals that have previously been placed in this slot.

The memory archive is updated every generation and after processing all individuals, the updated archive is used to initialize new individuals. For each new required, we pick a slot $S$ at random and calculate its distribution probability $P_{S}$ according to equation: $P_{S}=e^{\left(-N_{S}\right)}$ where $N_{S}$ is the value matching the slot key in the archive or zero if the slot does not yet belong to the archive. Finally, the calculated probability $P_{S}$ is compared to a uniformly distributed random number, $r$ and if $P_{S}>r$ then a new individual is initialized randomly in this specific slot. If not, another slot is picked at random, and the steps are repeated until the individual is initialized successfully. The Grid Diversity Operator (GDO is described in algorithm 1 .

The process of updating the memory archive and distributing new individuals continues until the algorithm terminates, at which point the final population is expected to be more diverse than simply using a random initialisation procedure.

\section{EXPERIMENTAL RESULTS}

GDO has been injected into Opt-aiNet algorithm [2] and both versions (with and without GDO) were run 25 times on a set of 10 unimodal optimization functions from the literature [3] (Ackley, Sphere, Rosenbrock function, GoldsteinPrice function, Booth, Bukin6, Matyas, Lévi13, Three-hump camel, Easom). The GDO variant was able to find the best solution in 7 out of the 10 functions in the test and with significantly less number of function evaluations as shown in figure 1 and it was confirmed by a Wilcoxon rank sum test.

A similar experiment were performed on 12 multimodal optimization functions from the literature [4] (Bird, Vincent, 

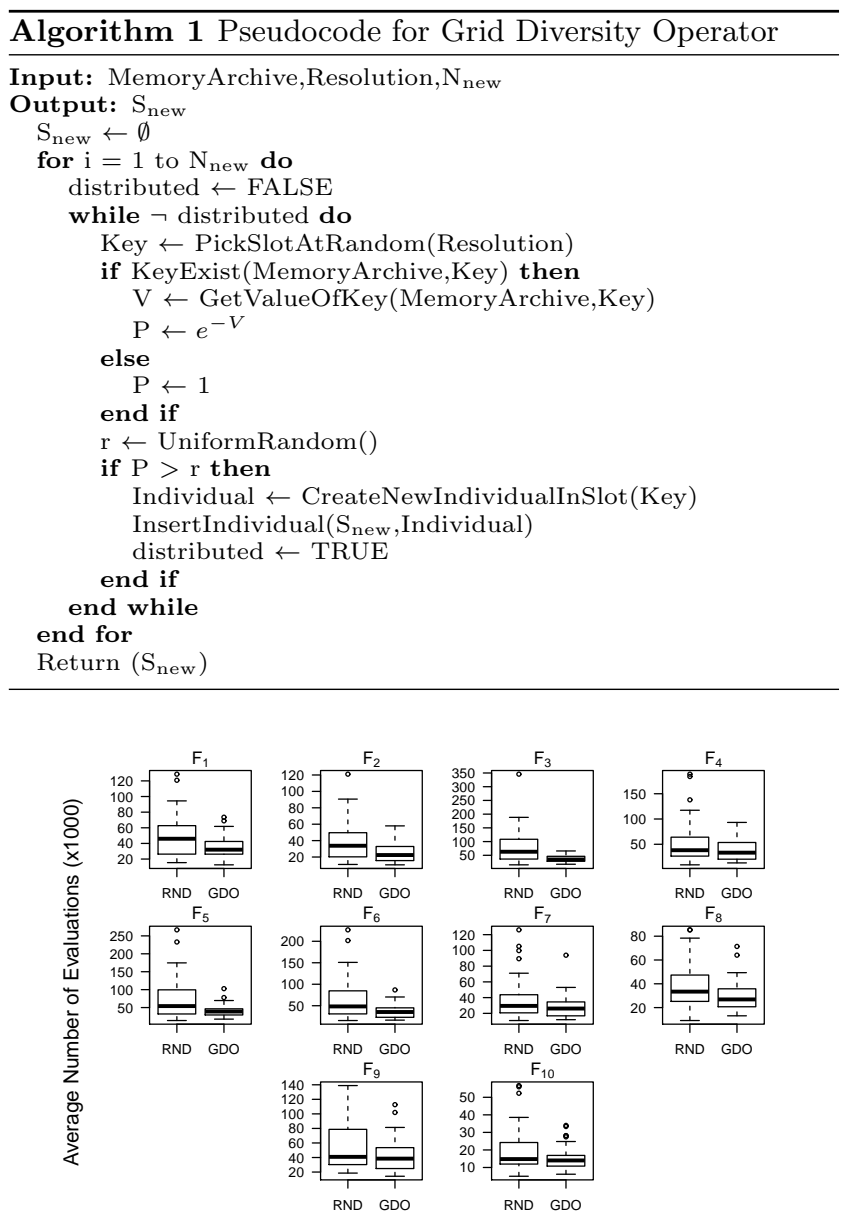

Figure 1: Graph comparing number of function evaluations between the variant with random initialization (RND) and GDO version

Roots, Hilly, Rastrigin, Himmemlblau, Foxholes, Guichif4, HolderTable, Rastrigin49, Schwefel) and the results are shown in figures 2 and 3 . The GDO version was able to find more optima than normal opt-aiNet in eight functions. The average number of function evaluations however was significantly lower with GDO than without it for all functions, according to a Wilcoxon rank sum test, showing improved performance on 11 functions out of the 12 .

\section{CONCLUSION}

The GDO operator was shown to achieve effective exploration through testing on both unimodal and multi-modal benchmarks when incorporated within opt-ainet. When compared to the original algorithm it was shown to be an order of magnitude faster at converging, and at least as good (and occasionally better) at finding peaks on the majority of functions. Although we have only provided results obtained by incorporating the operator into op-aiNet, the operator is by no means restricted to this function. It can be incorporated into any algorithm that includes an infusion step, e.g. the Saw-Tooth algorithm of [5].

\section{REFERENCES}

